\title{
XMM Observations of Abundances in the Intracluster Medium
}

\author{
Kyoko Matsushita \\ Tokyo University of Science, Japan \\ Yasushi Ikebe $^{1}$, Alexis Finoguenov ${ }^{2}$, Hans Böhringer ${ }^{2}$ \\ ${ }^{1}$ University of Maryland, USA ${ }^{2}$ Max-Planck-Institut fuer \\ Extraterrestrische Physik, Germany
}

\begin{abstract}
Based on XMM-Newton observations of M 87 and the Centaurus cluster, abundance profiles of various elements of the intracluster medium (ICM) are derived. The abundances of $\mathrm{Si}$ and Fe show strong decreasing gradients. In contrast, the $\mathrm{O}$ and $\mathrm{Mg}$ abundances are about half of the $\mathrm{Si}$ abundance at the center.

From the gas mass to stellar mass ratio and the comparison of $\mathrm{Mg}$ abundance with the stellar metallicity, the stellar mass loss from the central galaxies is indicated to be the main source of gas in the very central region of the clusters.

The observed $\mathrm{O}, \mathrm{Si}$ and $\mathrm{Fe}$ abundance pattern determines the contribution of supernova (SN) Ia and SN II, with the abundance pattern of ejecta of SN Ia. Most of the Si and Fe of the ICM in the central region of the clusters comes from SN Ia which occured in the central galaxies. In order to explain the observed $\mathrm{O} / \mathrm{Si}$ ratio of a half solar, SN Ia products should have similar abundances of $\mathrm{Si}$ and $\mathrm{Fe}$, which may reflect dimmer SN Ia observed in old stellar systems.
\end{abstract}

\section{Introduction}

The intracluster medium (ICM) contains a large amount of metals, which are mainly synthesized in early-type galaxies (e.g. Arnaud et al. 1992; Renzini et al. 1993). Thus, abundances of the metals are tracers of chemical evolution in galaxies and clusters of galaxies.

Based on the $\mathrm{Si} / \mathrm{Fe}$ ratio observed with ASCA, a discussion on contributions from SN Ia and SN II to the metals has commenced. In a previous nucleosynthesis model of SN Ia, the Fe abundance is much larger than the Si abundance in the ejecta of SN Ia (W7 model; Nomoto et al. 1984). Observations of metal poor Galactic stars indicate that average products of SN II have a factor of 2-3 larger abundance of $\alpha$-elements than Fe (e.g. Edvardsson et al. 1993; Nissen et al. 1994), although this ratio may depend on the initial mass function (IMF) of stars. In addition to the $\mathrm{Si}$ and Fe abundances, the XMM-Newton observatory enables us to obtain $\alpha$-element abundances such as for $\mathrm{O}$ and $\mathrm{Mg}$, which are not synthesized by SN Ia. 


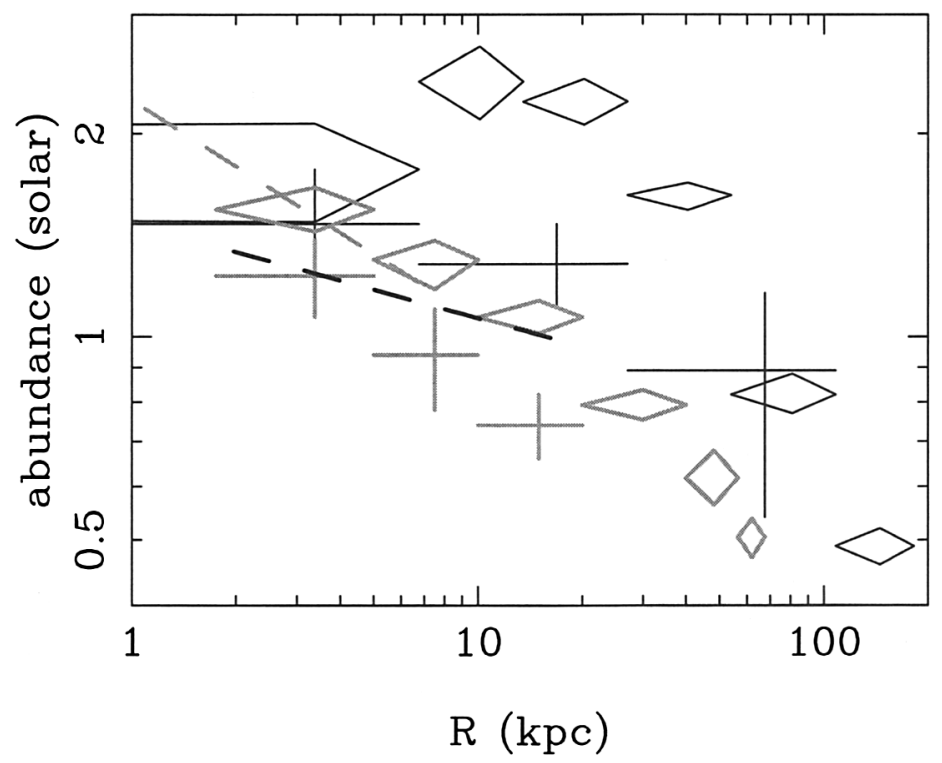

Figure 1. Abundance profiles of Fe (diamonds) and $\mathrm{Mg}$ (crosses) of M 87 (gray) and the Centaurus cluster (black). The dashed line represents the stellar metallicity derived from $\mathrm{Mg}_{2}$ index (Kobayashi \& Arimoto 2000).

In this paper, abundances of $\mathrm{O}, \mathrm{Mg}, \mathrm{Si}$, and $\mathrm{Fe}$ of $\mathrm{M} 87$ and the Centaurus cluster are discussed. We adopt for the solar abundances the values given by Feldman (1992), where the solar Fe abundance relative to $\mathrm{H}$ is $3.24 \times 10^{-5}$ in number.

\section{Observation}

M 87 was observed with XMM-Newton on June 19th, 2000. The effective exposures of the EPN and the EMOS are $30 \mathrm{ks}$ and $40 \mathrm{ks}$, respectively. The Centaurus cluster was observed on January 3rd, 2002. The effective exposures of the EPN and the EMOS are $29 \mathrm{ks}$ and $32 \mathrm{ks}$, The details of the analysis of background subtraction, vignetting correction and deprojection technique are described in Matsushita et al. (2002, 2003a, 2003b). When accumulating spectra of M 87, we used a spatial filter, excluding regions with soft emission around radio structures (Böhringer et al. 1995, Belsole et al. 2001, Matsushita et al. 2002).

\section{Contribution of gas from the $\mathrm{cD}$ galaxies}

The derived Fe abundances of the two clusters show strong negative gradients (Figure 1). However, that of the Centaurus cluster is systematically higher than that of $\mathrm{M} 87$ by nearly a factor of 2 at the same radii. 


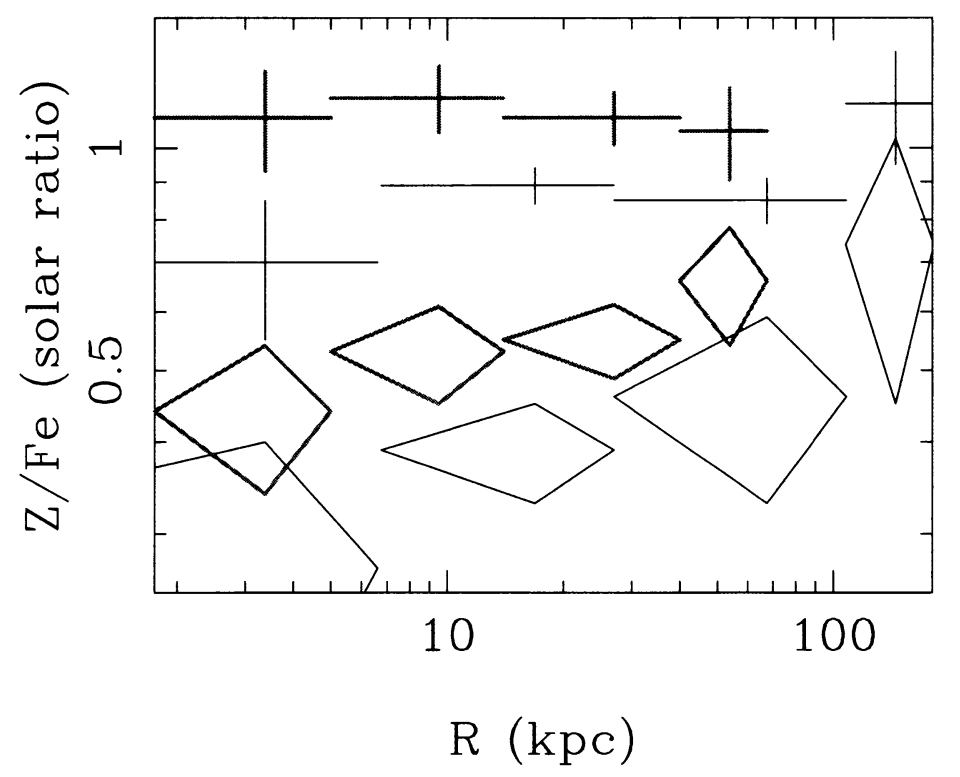

Figure 2. The $\mathrm{Si} / \mathrm{Fe}$ ratio (crosses) and $\mathrm{O} / \mathrm{Fe}$ ratio (diamonds) of $\mathrm{M}$ 87 (gray) and the Centaurus cluster (black).

The $\mathrm{Mg}$ abundances of $\mathrm{M} 87$ and the Centaurus cluster are derived through spectral fitting with the APEC model (Smith et al. 2001). Figure 1 also shows the observed $\mathrm{Mg}$ profiles with the stellar metallicity profiles from the $\mathrm{Mg}_{2}$ index (Kobayashi \& Arimoto 1999). The Mg abundance profiles of the ICM are consistent within $20 \sim 30 \%$ with the stellar metallicity profile at the same radius. Since we are comparing abundances in two distinct media, stars and ISM, which could have very different histories, the abundance results do not have to agree in general. But this agreement indicates that the ICM in this region should be dominated by the accumulation of gas lost from the central galaxy and contradicts the standard cooling flow model, consistent with the recent finding of the missing of the cooling gas (e.g. Makishima et al. 2002; Tamura et al. 2002; Böhringer et al. 2002; Matsushita et al. 2002). From the standard cooling flow model, the mass deposition rates of the Centaurus cluster and M 87 within 26 $\mathrm{kpc}$ and $10 \mathrm{kpc}$ respectively, are determined to be $15 M_{\odot}$ and $4 M_{\odot}$, respectively, (Allen and Fabian 1994; Matsushita et al. 2002), using a Hubble constant of 70 $\mathrm{Mpc} / \mathrm{km} / \mathrm{s}$. These values are much larger than the stellar mass loss rate within the radius, $\sim 0.4 M_{\odot}$ and $\sim 1 \odot$ for $\mathrm{M} 87$ and the $\mathrm{cD}$ of the Centaurus cluster, respectively. Therefore, if a cooling flow with this mass deposition rate exists, the fraction of gas from the central galaxy must be low, and the central abundance of the Centaurus cluster should be smaller than that of M 87. However, the observed result is the opposite.

Considering the gas mass and stellar mass loss rate, most of the $\mathrm{Si}$ and $\mathrm{Fe}$ at the center of M 87 come from present SN Ia in M 87 in the last few Gyr. While those in the center of the Centaurus cluster are accumulated at least over several 


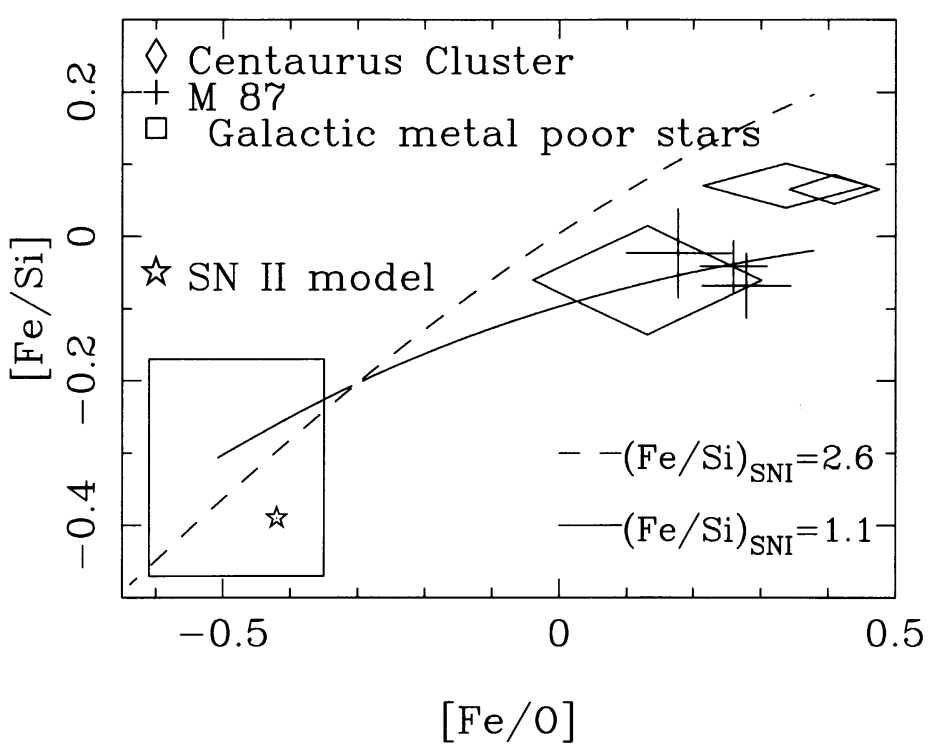

Figure 3. $\quad[\mathrm{Fe} / \mathrm{Si}]$ of the ICM of the Centaurus cluster (diamonds) and M 87 (crosses; Matsushita et al. 2003a) are plotted against [Fe/O]. The average value of Galactic metal poor stars (Clementini et al. 1999; open square) and the abundance ratio of SN II model using the nucleosynthesis model (asterisk) derived in Nomoto et al. (1997), assuming Salpeter's IMF (Iwamoto et al. 1999). The solid line and dashed line represent the relation of the abundance pattern synthesized by SN Ia with $\mathrm{Fe} / \mathrm{Si}=1.1$ (the best fit relation of $\mathrm{M} 87$ ) and $\mathrm{Fe} / \mathrm{Si}=2.6$ (W7 ratio; Nomoto et al. 1984), respectively.

Gyr. In order to explain the higher Fe abundance in the Centaurus cluster, the Fe production rate should have been higher in the past.

\section{Abundance ratios of $\mathrm{O} / \mathrm{Si} / \mathrm{Fe}$ and abundance pattern of $\mathrm{SN}$ Ia}

Figure 2 shows the observed $\mathrm{Si} / \mathrm{Fe}$ and $\mathrm{O} / \mathrm{Fe}$ ratios of the two clusters. The $\mathrm{Si} / \mathrm{Fe}$ ratio is determined to be close to unity. For $\mathrm{M} 87$, the $\mathrm{Si} / \mathrm{Fe}$ ratio is $\sim 1.1$ solar, and for the Centaurus cluster, it is $\sim 0.9$ solar ratio. In contrast, the $\mathrm{O} / \mathrm{Fe}$ ratio is less than 0.5 solar at the center and increases with radius.

Figure 3 summarizes the abundance pattern of $\mathrm{O}, \mathrm{Si}$, and $\mathrm{Fe}$ of the ICM. Although the abundance pattern of ejecta of SN II may differ between early-type and late-type galaxies, and that of SN Ia also may not be a constant (Umeda et al. 1999; Finoguenov et al. 2002), for a first attempt we have assumed $(\mathrm{Fe} / \mathrm{Si})_{\mathrm{SNIa}},(\mathrm{Si} / \mathrm{O})_{\mathrm{SNII}}$, and $(\mathrm{Fe} / \mathrm{O})_{\mathrm{SNII}}$ to be constants. Here, $(\mathrm{Fe} / \mathrm{Si})_{\mathrm{SNIa}}$ is the $\mathrm{Fe} / \mathrm{Si}$ ratio of ejecta of $\mathrm{SN} \mathrm{Ia}$, and $(\mathrm{Si} / \mathrm{O})_{\mathrm{SNII}}$ and $(\mathrm{Fe} / \mathrm{O})_{\mathrm{SNII}}$ are the $\mathrm{Si} / \mathrm{O}$ ratio and the $\mathrm{Fe} / \mathrm{O}$ ratio of the ejecta of $\mathrm{SN}$ II, respectively.

The classical deflagration model of SN Ia, W7 (Nomoto et al. 1984), expects the $\mathrm{Fe} / \mathrm{Si}$ ratio of 2.6 solar ratio. When we adopt the abundance pattern of the 
Galactic metal poor stars by Clementini et al. (1999) as that of SN II, (Fe/Si) SNIa of M 87 is determined to be $\sim 1$ solar. That of the center of the Centaurus cluster is slightly larger than M 87, but still smaller than the W7 ratio. Thus, these values are much smaller than W7 model (Nomoto et al. 1984), and in the range of the ratios derived from the WDD models (Iwamoto etal. 1999), which considers slow deflagration.

The light curves of observed SN Ia are not identical but display a considerable variation (e.g. Hamuy et al. 1996). In SN Ia, the mass of synthesized $\mathrm{Ni}^{56}$ determines the luminosity of each SN. Since the mass of the progenitor should be constant at $1.4 M_{\odot}$, the ratio of mass of intermediate group elements from $\mathrm{Si}$ to $\mathrm{Ca}$, to the mass of $\mathrm{Fe}$ and $\mathrm{Ni}$, should depend on the luminosity of SN Ia. The observed luminosity of SN Ia correlates with the type of the host galaxy, and is suggested to be related to the age of the system; SNe Ia in old stellar system may have smaller luminosities (Iwanov et al. 2000), and hence are suggested to yield a smaller Fe/Si ratio (e.g. Umeda et al. 1999).

As discussed in Finoguenov et al. (2002) and Matsushita et al. (2003a;2003b) the smaller $\mathrm{Fe} / \mathrm{Si}$ ratio observed for the ICM around M 87 and the Centaurus cluster may reflect the fact that $\mathrm{M} 87$ and the $\mathrm{cD}$ galaxy of the Centaurus cluster are old stellar systems.

\section{References}

Allen, S. W., \& Fabian, A.C., 1994, MNRAS, 269, 409

Arnaud M., Rothenflug R., Boulade O., et al. 1992, A\&A, 254, 49

Belsole E., Sauvageot, J.L., Böhringer, H., et al. 2001, A\&A, 365, L188

Böhringer, H., Belsole, E., Kennea, J., et al. 2001, A\&A, 365, L181

Böhringer, H., Matsushita, K., Churazov, E., Ikebe, Y., \& Chen, Y., 2002, A\&A, 382,804

Clementini, G., Gratton, R.G., Carretta, E. et al., 1999, MNRAS, 302, 22

Edvardsson, E., Andersen, J., Gustafsson B., et al. A\&A,1993, 275, 101

Feldman, U., 1992, Physica Scripta 46, 202

Finoguenov A., Matsushita, K., Böhringer, H., et al. 2002, A\&A, 381, 21

Hamuy, M., Philips, M.M., Suntzeff, N.B., et al. 1996, AJ112, 2438

Iwamoto, K., Brachwitz, F., Nomoto, K., et al. 1999, ApJS, 125,439

Iwanov, V., Hamuy, M., \& Pinto, P.A., 2000, ApJ, 542,588

Kobayashi, C., \& Arimoto, N., 1999, ApJ, 527, 573

Nissen, P.E., Gustafsson, B., Edvardsson, B., et al. 1994, A\&A, 285, 440

Nomoto, K., Thielemann, F-K., \& Wheeler, J.C., 1984, ApJ, 279, 23

Makishima K., Ezawa H., Fukazawa Y., et al. 2001, PASJ, 53, 401

Matsushita, K., Belsole, E., Finoguenov, A., \& Böhringer, H., 2002, A\&A, 386, 77

Matsushita K., Finoguenov A., Böhringer H., 2003a, A\&A, 401, 443

Matsushita K., Böhringer H., Takahashi I., \& Ikebe Y., 2003b, submitted to $\mathrm{A} \& \mathrm{~A}$ 
Renzini A., Ciotti, L., D'Ercole, A., \& Pellegrini, S. 1993, ApJ, 419, 52

Smith, R.K., Brickhouse, N.S., Liedahl,D.A., \& Raymond, J.S., 2001, ApJ, 556, 91

Tamura T., et al. 2001, A\&A, 365, L87

Umeda H., Nomoto K., \& Kobayashi C. 1999, ApJ, 522, L43 